\title{
Numerical simulations for the optimisation of ventilation system designed for wine cellars
}

\author{
Enrica Santolini, Alberto Barbaresi, Daniele Torreggiani, Patrizia Tassinari \\ Department of Agricultural and Food Sciences, University of Bologna, Italy
}

\begin{abstract}
The wine-ageing process is one of the most important phases of the wine production and it can be considerably affected by the micro-climatic conditions inside the ageing rooms. Underground wine cellars in small-medium wineries are designed with natural ventilation systems, able to maintain optimal indoor condition. However, critical factors emerge, such as mold growth or wine evapo-transpiration, where ventilation proved to be poorly designed, insufficient in the first case or excessive in the second one. The zones around the wooden barrels proved to be the most sensitive and problematic. These areas are the most investigated in terms of temperature and humidity values but surprisingly not in terms of air velocity. In this paper, a ventilation system has been designed and optimised to support the lack of ventilation, by means of computational fluid dynamics modelling. Eight configurations have been performed and analysed, identifying the best two according to the air velocity range. Specific parameters have been defined to appreciate the application limits of each configuration. These parameters can be used as reference for system design in similar studies and applications and can help scholars and professionals to identify the optimal configurations for the implementation and proper placement of the system inside a cellar.
\end{abstract}

Correspondence: Enrica Santolini, Department of Agricultural and Food Sciences, University of Bologna, via Giuseppe Fanin 48, 40127 Bologna, Italy.

E-mail: enrica.santolini2@unibo.it

Key words: Indoor air; wine cellar; computational fluid dynamic modelling; natural ventilation; perforated tube; numerical simulations.

See online Appendix for additional Figures.

Received for publication: 16 March 2019.

Accepted for publication: 16 July 2019.

CC Copyright: the Author(s), 2019

Licensee PAGEPress, Italy

Journal of Agricultural Engineering 2019; L:952

doi:10.4081/jae.2019.952

This article is distributed under the terms of the Creative Commons Attribution Noncommercial License (by-nc 4.0) which permits any noncommercial use, distribution, and reproduction in any medium, provided the original author(s) and source are credited.

\section{Introduction}

The importance of internal environmental conditions in a wine cellar is well known and investigated (Mazarrón and Cañas, 2009; Geyrhofer et al., 2011; Benni et al., 2013; Barbaresi et al., 2015b). The winemaking process consists in several steps and one of the most important and interesting is the wine ageing. In this step, temperature and humidity play a fundamental role, since the quality of the final products is strongly affected by environmental conditions (Boulton et al., 1999). Since the past centuries, the research has been devoted to the definition of ideal room conditions for wine-ageing process performed in wooden barrels (Benni et al., 2013). In literature, even though different suitable ranges have been defined for different wines, it is assessed that the wine should be generally kept at temperatures ranging from $9^{\circ} \mathrm{C}$ to $20^{\circ} \mathrm{C}$. Vogt (1971) defined that an optimal thermal excursion should be smaller than $6^{\circ} \mathrm{C}$ and (Togores, 2003) considered that the relative humidity should be higher than $70 \%$, in order to ensure a quality ageing and to prevent excessive wine loss due to evaporation. The wine losses have been related to a combination of air temperature and humidity (Negrè and Françot, 1965) showed how high temperatures, couple with low relative humidity, can increase the wine evaporation, as for example $18^{\circ} \mathrm{C}$, with $45 \%$, produces wine losses of $4-7 \%$ in volume per year. Ruiz De Adana et al. (2005) developed a mathematical model that correlates wine losses to the room conditions, thus quantifying how air velocity, temperature and humidity can affect wine evaporation (Barbaresi et al., 2014). This model considers the air velocity over the barrels, which could modify the wood surface emission coefficient (Avramidis and Siau, 1987) and therefore the evaporation through the barrel staves, suggesting, moreover, that low air velocity values could prevent excessive wine losses.

Furthermore, Simeray et al. (2001) explained that relative humidity could favour mold and other fungi formation, which may potentially contaminate products or also affect wine quality and organoleptic properties. Regarding this, Ocón et al. (2011) showed that the ventilation could be identified as a fundamental factor to reduce the mold presence in the air, since decreasing the possibility of mold proliferation on both walls and wooden surface of the barrels.

Moreover, the scientific literature highlights the relevant role of the air velocity - beside temperature and humidity - in the wine-ageing process. On their research, Ruiz De Adana et al. (2005) pointed out the role of air velocity in the wine losses in ageing facilities. So far, despite its importance, optimal values of air velocity close to barrels have not been identified in scientific research. However, a few papers report that the natural ventilation inside cellars usually leads to air velocity around $0.3-0.4 \mathrm{~m} / \mathrm{s}$ (Geyrhofer et al., 2011). It is important to note that, due to typical cellar layouts (small openings and barrels located close to the 
walls) the natural ventilation inside the cellars could be uncontrolled, insufficient or could create airflow distribution characterised by stagnation zones, frequently close to the wooden barrels, entailing that proper air velocity and distribution can ease a correct wine ageing, reducing risks of mould formation and wine losses. As described, the indoor environment in wine cellars is a delicate matter and the required conditions can hardly be maintained, in particular, in non-conditioned rooms where zones with different thermal conditions can be found even in open space rooms (Barbaresi et al., 2015a). Under this light proper ventilation can ease to create the homogeneity of thermal condition in the room. For this reason, it is reasonable to think that computational fluid dynamics (CFD) simulations can give an important contribution to design cellar layouts or systems. Despite the significant role of ventilation in the environmental condition in wine cellar, the application of CFD for improving the ventilation in this sector is really rare, compared to other sector such as livestock farming (Rong et al., 2016) or greenhouse sector (Bartzanas et al., 2013; Santolini et al., 2018). Recently, De Rosis et al. (2014) has conducted a numerical study of airflows in a wine aging-room, using a Lattice-Boltzamann method. This study underlines how barrels are differently involved in airflows and consequently the identification of the most emblematic points for air measurements.

This paper aims at studying and designing a forced air ventilation system, in support to the natural ventilation specifically optimised for wine ageing rooms. This system, designed to be efficient and cost effective for small producers, is created using PVC tube and PC fans. Similar systems are commonly applied in other sectors and just few studies investigated their application in agro-food sector, as example Mondaca and Choi (2017) realised with and validated a CFD model capable of replicating the flow distribution created by a positive-pressure polyethylene tube ventilation system, designed for a livestock (Wang et al., 2018). In this paper, a 3D model of the ventilation system has been carried out and validated based on an experimental system. This system has been also optimised to give the proper air velocities, with the future purpose of its implementation and test in a wine cellar.

\section{Materials and methods}

The natural ventilation in a wine cellar is usually obtained thanks to small windows and different outlets in the room. As already presented in the Introduction, the ventilation presents various issues. Then, a supportive ventilation system has been designed to be placed in a wine cellar, in addition to the existing ventilation system. This system has been designed to be suitable for small or medium wine producers.

\section{Case study}

An underground wine-ageing room of an Italian winegrowing and producing farm has been chosen as case study (farm winery with average production of 2500-3000 hL/year, representative size of a small-medium producer). The farm is in the eastern part of the Emilia-Romagna Region, where viticulture and fruit farming are widespread and consolidated activities (Barbaresi et al., 2015b). Figure A.1 shows the wine cellar room from outside (picture on the left) and from inside (picture on the right). The room (shown in Figure A.1) is a northeast oriented parallelepiped, $9.80 \mathrm{~m}$ long, $5.20 \mathrm{~m}$ wide and $2.60 \mathrm{~m}$ high and it has window located on the northeast wall, a door on the north-west wall. It can contain up to 45 French barrels, called barriques, $89 \mathrm{~cm}$ long, and diameter 59$71 \mathrm{~cm}$ each. The barrels are placed close to the walls and few of them also in the middle of the room.

The four walls and the floor are in contact with the ground while the ceiling is in contact with a conditioned room. The cellar is naturally ventilated but it had showed several inconveniences for the wine conservation. The air velocity could be too low around the barriques, raising in this way the possibilities of mold formation, or even stagnation areas not reached by natural ventilation, as highlighted by the work made by De Rosis et al. (2014). Currently, these problems are addressed with periodic and systematic forced air-changes in the room. Despite its proved effectiveness, this solution requires personnel, time and specific equipment; furthermore, the system set up is defined by the personnel experience only, therefore a more effective solution, based on a specific study, is required.

\section{Fundamental computational fluid dynamics equations}

Computational fluid dynamics is based on the governing fluid dynamics equations (continuity, momentum and energy). The equations obtained directly from the volume or fixed element in space are known as conservative form. While the equations which move with the fluid element, are called as non-conservative form (De la Torre-Gea et al., 2011). The fluid flow can be modelled by the Navier-Stokes (N-S) equations that are time and space dependent. Non-linear convective terms are present in the N-S equation that raises the difficulties on the equation resolution, such as the pressure-gradient term. In fact, if expressed as a function of velocity (which is a time-and-space-dependent variable), the pressure gradient becomes non-linear and non-local. To solve the N-S equations in turbulent flow, it is possible to resort to turbulence models. These models solve equations for some main quantities, e.g. $\mathrm{U}, \overline{\mathrm{u}}_{i} \overline{\mathrm{u}}_{j}$ or $\varepsilon$. Reynolds-averaged Navier-Stokes are part of this family, since they involve the solution of the Reynolds equation to identify the mean velocity field. The Reynolds-averaged form of basic equations is obtained when the instantaneous value of a quantity is expressed with two components: a time-averaged component, independent from time, and a time-varying component, for which the time average is equal to zero. The Navier-Stokes equation, formulated for $x$ direction and with Boussinesq approximation, has this form:

$$
\rho\left(\overline{\mathrm{u}} \frac{\partial \overline{\mathrm{u}}}{\partial x}+\bar{v} \frac{\partial \bar{u}}{\partial y}+\bar{w} \frac{\partial \bar{u}}{\partial z}\right)=-\frac{\partial p}{\partial x}+\left(\mu+\mu_{t}\right)\left(\frac{\partial^{2} \bar{u}}{\partial x^{2}}+\frac{\partial^{2} \bar{u}}{\partial y^{2}}+\frac{\partial^{2} \bar{u}}{\partial z^{2}}\right)
$$

The mean field of physical quantities is calculated, leaving terms of upper orders to second one. An expression, named enclosure, for the Reynolds stress term is needed in the Navier-Stokes equation. Several possible models are available and one of them is the realisable $k-\varepsilon$ model, a variation of the standard $k-\varepsilon$ model. The $k$ is the kinetic energy per unit mass of the turbulent fluctuations and $\varepsilon$ is the dissipation rate. This model is a semi-empirical model and the derivation of the model equations relies on phenomenological considerations and empiricism. $K$ - $\varepsilon$ realisable model is different from the $k-\varepsilon$ standard model in two ways (Shih et al., 1995): i) contains an alternative formulation for the turbulent viscosity; ii) considers a modified transport formulation for the dissipation rate: $\varepsilon$, which has been derived from an exact equation for the transport of the mean-square vorticity fluctuation (Eqs. 2 and 3). 
The transport equations of the model are:

$\frac{\partial}{\partial t}(\rho \kappa)+\frac{\partial}{\partial x_{i}}\left(\rho \kappa u_{j}\right)=\frac{\partial}{\partial x_{j}}\left[\left(\mu+\frac{\mu_{t}}{\sigma_{k}} \frac{\partial \kappa}{\partial x_{j}}\right)\right]+G_{k}+G_{b}-\rho \epsilon-Y_{M}+S_{k}$,

and

$$
\begin{aligned}
\frac{\partial}{\partial t}(\rho \epsilon)+\frac{\partial}{\partial x_{i}} & \left(\rho \epsilon u_{j}\right) \\
& =\frac{\partial}{\partial x_{j}}\left[\left(\mu+\frac{\mu_{t}}{\sigma_{\epsilon}} \frac{\partial \epsilon}{\partial x_{j}}\right)\right]+\rho C_{1} S_{\epsilon}-\rho C_{2} \frac{\epsilon^{2}}{k+\sqrt{v \epsilon}}+C_{1 \epsilon} \frac{\epsilon}{k} C_{3 \epsilon} G_{b}+S_{\epsilon}
\end{aligned}
$$

where $C_{1}=\max \left[0.43, \frac{\eta}{\eta+5}\right], \eta=S \frac{k}{\epsilon}$ and $S=\sqrt{S_{i j} S_{i j}}$.

$C_{1}$ is $1.44, C_{2}$ is $1.9, \sigma_{\varepsilon}$ and $\sigma_{\kappa}$ are 1.2 and 1 respectively. In AnsysFluent, the software used in this work, $C_{3 \varepsilon}$ is not defined but it is calculated according to this expression (Fluent, 2006):

$$
C_{3 \epsilon}=\tanh \left|\frac{v}{u}\right|
$$

where $v$ is the component of the flow velocity parallel to the gravitational vector and $\mathrm{u}$ is the component of the flow velocity perpendicular to the gravitational vector. In particular, it is defined realis- able because the model satisfies specific mathematical constraints on the Reynolds stresses, consistent with the physics of turbulent flows. For the $\mathrm{k}-\varepsilon$ standard, this definition is not applicable. A significant benefit of the realisable $\mathrm{k}-\varepsilon$ model is that exhibits more accuracy to predict the spreading rate of both planar and round jets. It is also likely to provide greater performance than standard $\mathrm{k}-\varepsilon$ model in simulation with flows involving rotation, such as the air jet coming from a fan, as in this case.

\section{D model and grid convergence}

Tube ventilation is a solution designed to gently introduce fresh air into enclosed environments with the aim to avoid uncontrolled drafts. For this reason, it has been considered as an effective solution in non-residential buildings, including agricultural buildings as calf barns and other animal rearing facilities, such as poultry and swine as well as greenhouses, commercial buildings, industrial settings, etc. However, its application in wine cellars has been poorly investigated. In this case, this system has been considered to improve the climatic conditions around the barrels; this entails geometrical constraints to the ventilation system, due to the space availability between the barrels and the wall. Hence, to be placed behind the barriques, the tube cannot have a diameter greater than $20 \mathrm{~cm}$ and be longer than $4.80 \mathrm{~m}$, as visible in Figure A.2. The system has been designed thinking to materials that are either to find and not expensive. For these reasons, a PVC tube of $0.12 \mathrm{~m}$ of diameter, $2 \mathrm{~m}$ long and a PC fan of $220 \mathrm{~V} \mathrm{DC}$, with proper connections have been used. In the tube, 5-mm-diameter holes have been

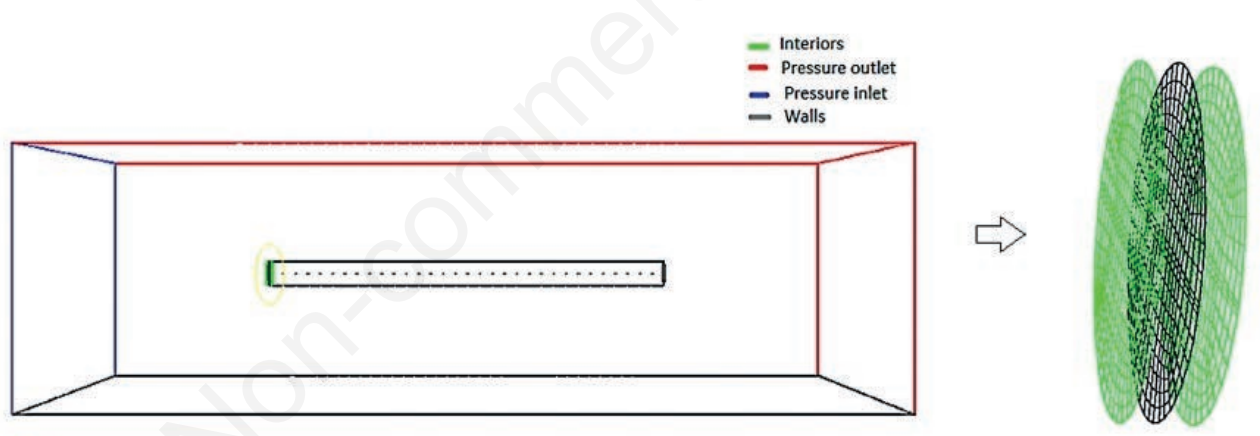

(A)

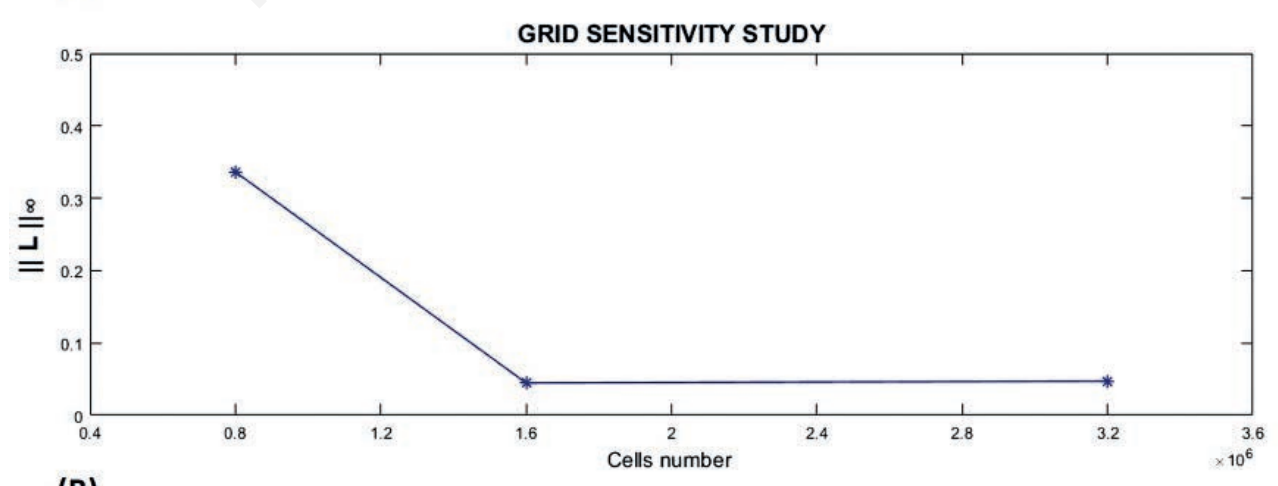

(B)

Figure 1. The 3D model of the case, with outlined boundary surfaces specification, is presented in the top figure (A). A yellow ring highlights the fan and its magnification has been shown at the right. The three visible surfaces are the two interior surfaces, in green, and the fan surface in black. In the bottom image, (B), the grid convergence study results are reported, for the four different meshes, by the calculation of $\|\mathrm{L}\| \infty$. 
created with a drill and then the system has been tested (see Figure A.3 in the Appendix). Air velocity measurements have been performed with a hot wire anemometer (Delta Ohm with an uncertainty of $0.01 \mathrm{~m} / \mathrm{s}$ ) at $0.40 \mathrm{~m}$ from tube to verify the range of velocity reachable at that distance. The optimal target range of velocity, based on (Geyrhofer et al., 2011), has been defined up to $0.40 \mathrm{~m} / \mathrm{s}$ to $1 \mathrm{~m} / \mathrm{s}$. These measurements have been used also to validate the CFD model. The geometrical model has been developed with Autodesk Inventor. The global dimensions of the domain are $3 \mathrm{~m}$ wide, $4.025 \mathrm{~m}$ long and $1.2 \mathrm{~m}$ height. In particular, the fan has been modelled as a surface inserted into a solid body and has been designed as an internal surface of a small cylinder, its closing surfaces have been defined as interiors. The small cylinder has been created with a base of $12.5 \mathrm{~cm}$ of diameter and a height of $2.5 \mathrm{~cm}$. It has been created a surface that cuts in two equal part the cylinder, in order to model that surface as a fan in Ansys-Fluent, simplifying the complete 3D design of the fan structure. Globally boundary conditions of these cases have been defined as visible in Figure 1. The fan has been already defined with initial velocity of 2450 rpm for the validation process, which is the characteristic velocity of the fan. It has been configured with a static pressure of $45 \mathrm{~Pa}$ derived from the characteristic of the fan, the centre of rotation exactly matches the centre of the surface, with a diameter of the internal body equal to half of the fan diameter, $0.06 \mathrm{~m}$. On this model, four different grids with cells number from $8 \times 10^{5}$ to the finest one of $6.4 \times 10^{6}$ have been performed. As turbulence model, the $\mathrm{k}-\varepsilon$ realisable has been applied and the Second order up-winding discretisation schemes have been used for pressure, momentum, $k$ and $\varepsilon$ to increase the accuracy and to reduce numerical diffusion.

The SIMPLE scheme has been used for the pressure-velocity coupling. FLUENT uses an iterative method to solve the algebraic system of equations. A termination criterion of $10^{-6}$ for continuity and $10^{-5}$ has been used for all the other field variables. Then, a convergence study has been performed as presented in Figure 1. Ten velocity profiles at several distances from the tube have been considered and ILL $\|_{\infty}$ (see Eq. 5) (Magnini et al., 2016; Santolini et al., 2018) has been computed progressively between two different meshes, with increasing number of cells:

$$
\|L\|_{\infty}=\max \left|v_{a}-v_{b}\right|
$$

The resulting mesh, chosen for the simulations, has been made by $3.2 \times 10^{6}$ cells.

\section{Model validation}

In order to validate the presented model, the system underwent measurements of air velocity. A series of measures has been taken at $40 \mathrm{~cm}$ from the system, in four different positions along the tube, as previously mentioned. This distance has been considered a representative distance of the barrels from the tube once placed in the wine cellar, according to the case-study cellar layout. The measurements have been performed in correspondence with holes 3, 16, 19 and 28, as visible in Figure A.4. Each measurement has been performed for 2 minutes, recording data within $2 \mathrm{~s}$ time steps. In Figure 2, panel A on the left shows the air velocity profile at $40 \mathrm{~cm}$ from the tube, extrapolated from the simulation compared to the measured data. It is already visible that the numerical solution is significantly close to the real data. The simulation result shows an oscillation of the air velocity among all the profile that is characterised by a significant reduction at the centre of the tube. The air velocity reaches a minimum pick of $0.35 \mathrm{~m} / \mathrm{s}$ and a maximum pick of $0.68 \mathrm{~m} / \mathrm{s}$.

The comparison between the two trends shows that the result of simulation has been linearized as visible in the Figure $2 \mathrm{~B}$. Calculating the relative error between the measured data and the simulation results in the same positions, three on four errors are less than $10 \%$, precisely are $5 \%, 2 \%$ and $1 \%$, as reported in Table 1. Moreover, the root mean square error calculated based on the four measurements is equal to $0.008 \mathrm{~m} / \mathrm{s}$.

However, the measurement data collected at the beginning of the ventilation system, in the first point, shows the most relevant error compared to the simulated results. This can be due to the effect of the fan swirl effect, as explained in Mondaca and Choi 2017 , that could not be well appreciated by the simulation model.

(A)

Validation model
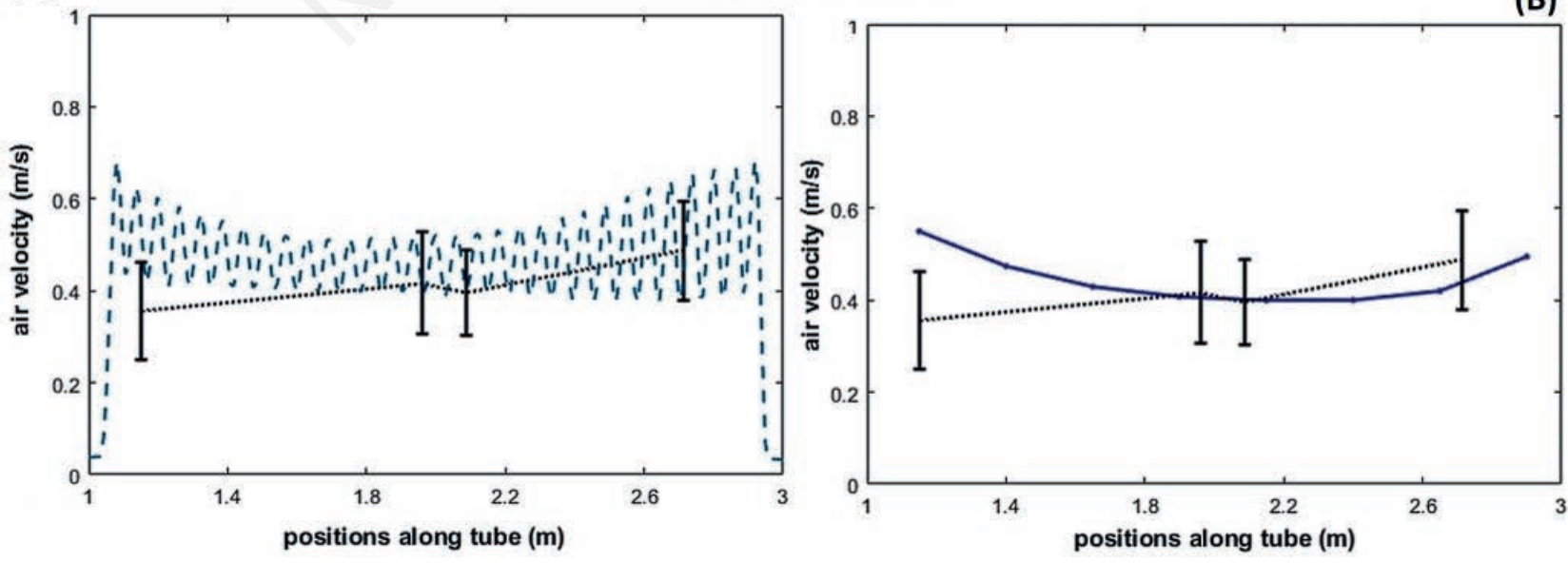

Figure 2. The figure shows the comparison between measured and simulated velocity magnitudes, collected at $40 \mathrm{~cm}$ from the tube. A) It shows the profile velocity from the model compared to the profile of collected data (dashed profile); B) it shows the measurements profile compared to the linearized profile of simulated results. 
However, the error is significantly limited and does not affect the validation of the model. For what said above, the differences between real and simulated data can be assessed as not significant and therefore the model is considered validated.

\section{System configurations}

The system used for the validation procedure is the first system design analysed in this paper. Then, other possible solutions have been considered, aiming to find the best configuration able to obtain the most homogeneous air velocity profile, with a magnitude settled in a range between $0.3 \mathrm{~m} / \mathrm{s}$ and $1 \mathrm{~m} / \mathrm{s}$, at $40 \mathrm{~cm}$ from the tube. Firstly, several preliminary simulation tests suggested enlarging the holes, maintaining the length of the tube and the $d / \varphi$ ratio equal to 12 . Then, the number of holes has been thinned out. Therefore, the new hole diameter has been set to $8 \mathrm{~mm}$ instead of $5 \mathrm{~mm}$, and new hole distance is set to $9.6 \mathrm{~cm}$. Taking into account the effect of the swirl of the fan - that can create high turbulence in the first part of the tube (Brundrett, 1990) and maintaining the holes diameters of 5 and $8 \mathrm{~mm}$ - the previous two configurations have been designed with an addition of $20 \mathrm{~cm}$ of tube spacing the fan from the first hole. Other four configurations, starting from the solution with $8 \mathrm{~mm}$ and $5 \mathrm{~mm}$ holes, have been created reducing the $d / \varphi$ ratio of one third or halved. In this way, the distance between holes has been decreased and consequently their number increased. The idea behind the present choice has been to obtain a reduction of the range of variation of the velocity magnitude.

Totally, eight different system set ups, summarised in Table 2, have been provided and analysed. The comparisons among them have been performed based on the idea that configuration (1) and (2) have been considered the starting set ups and the subsequent configuration should demonstrate to be an improvement of the first ones.

\section{Results}

The result analyses have been carried out first on the basic Configurations ((1) and (2)) and then progressively on the others.

The validated Configuration (1) has been firstly compared to the results obtained for Configuration (2). In Figure 3, the Configuration (1) shows a limited air velocity variation compared to Configuration (2), on the contrary, the latter presents a velocity variation between 0.2 and $0.8 \mathrm{~m} / \mathrm{s}$. Based on the averaged values, the air magnitude is higher than the Configuration (1) but it is still contained in the established range. On one hand, the solution with 8-mm holes (Configuration (2)) presents a more homogeneous magnitude. On the other hand, the $5 \mathrm{~mm}$ holes solution (Configuration (1)) shows an increasing air velocity in the first and last few holes. This fact has been linked to the turbulent nature of the flow and reasonably to the dependence on the swirl of the fan. Taking into account these results, it has expected in Configurations (3) and (4) to have results less affected by the fan rotation. On the contrary Configuration (3) shows an unexpected air velocity trend, visible in Figure 3. In fact, the air magnitude significantly decreases at the level of half tube, reaching values as low as $0.2 \mathrm{~m} / \mathrm{s}$. The trend of the air speed for Configuration (4) does not change compared to Configuration (2), despite the addiction of $20 \mathrm{~cm}$ of tube close to the fan. This does not affect the resulting velocity at $40 \mathrm{~cm}$ of distance from the tube, except for the peak at fan level, which was not present in the previous configurations. The trend variation of air velocity is similar to the starting tube set up (Configuration (1)). Air jets penetration distances are comparable in Configurations (2) and (4), as visible in Figure 4. Instead, the air jets penetration distances of Configuration (3) are significantly reduced compared to the starting Configuration (1) (Figure 4). As visible in Figure 4, for the most configurations, the profiles (black line in the figure) are placed in a zone where the air jets are clearly defined. Instead, a few centimetres further from the profile, the separation of between each single jet is no longer noticeable, identifying an area with a more homogeneous and mixed air velocity.

Nevertheless, the air jets of Configurations (1) and (3) is characterised by more restricted penetration distances. In particular, Configuration (3) presents a partial area, over the profile line, where there is no evident effect of the system (contour profiles are not present). In this area the air velocity is under the lower limit defined $(0.05 \mathrm{~m} / \mathrm{s})$. Hence, we can assume that this configuration definitely has a very limited area of action. However, considering the application of the system and the desired air velocity, the new configurations ( 3 and 4 ) return a performance not suitable for the cellar needs. In Figure 4, the contour map of all configurations (from (1) to (8)) are reported, showing the air velocity magnitude of the area around the system. Observing specifically the last two configurations $((7)$ and (8)), it is clear that the air jets, coming from the system, have a wide area of action in both cases. In fact, downstream of the pipe stagnation areas (air velocity equal to $0 \mathrm{~m} / \mathrm{s}$ ) are not detected, however some areas at low velocity $(0.05 \mathrm{~m} / \mathrm{s})$ are present in the results of Configuration (7) only. On the contrary, Configuration (8) shows larger effects compared to the others, presenting an area at air velocity higher than $0.4 \mathrm{~m} / \mathrm{s}$ from $40 \mathrm{~cm}$ up to the limit of the domain, which means over $1.45 \mathrm{~m}$ far from the pipe. Taking into account the velocity profile at $40 \mathrm{~cm}$ from the system, the previous comparison has been performed also between Configurations (1) and (5), (2) and (6), as shown in Figure 3. The Configurations (5) and (6) show significantly limited velocity variation, swinging around $0.6 \mathrm{~m} / \mathrm{s}$ for the Configuration (5), and between 0.6 and $0.8 \mathrm{~m} / \mathrm{s}$ for the Configuration (6). Configuration (5) presents also a wider initial variation of air speed, at the level of the first five holes, but still limited between 0.4 and $0.6 \mathrm{~m} / \mathrm{s}$.

Table 1. Numerical comparison between the measured data and the relative simulated results.

\begin{tabular}{lccc} 
Measurement & $V_{\text {measured }}(\mathrm{m} / \mathrm{s})$ & $V_{\text {simulated }}(\mathrm{m} / \mathrm{s})$ & Brror $(\mathrm{m} / \mathrm{s})$ \\
1 & 0.36 & 0.414 & 0.06 \\
2 & 0.42 & 0.433 & 0.02 \\
\hline 3 & 0.40 & 0.444 & 0.05 \\
4 & 0.49 & 0.477 & 0.01 \\
\hline
\end{tabular}

Table 2. Configurations of the tube analysed in this study: $\mathrm{N}$ is the number of holes; $L$ is the length of tube and $\varphi$ is the diameter of holes.

\begin{tabular}{llcll} 
Configurations & $N$ & $\varphi(\mathrm{mm})$ & $d / \varphi$ & $L(\mathrm{~m})$ \\
1 & 30 & 5 & 12 & 2 \\
2 & 19 & 8 & 12 & 2 \\
\hline 3 & 39 & 5 & 12 & 2.2 \\
4 & 19 & 8 & 12 & 2.2 \\
\hline 5 & 44 & 5 & 8 & 2 \\
6 & 27 & 8 & 8 & 2 \\
\hline 7 & 58 & 5 & 6 & 2 \\
8 & 35 & 8 & 6 & 2 \\
\hline
\end{tabular}


This effect remains an isolated episode in the profile and is still in the range of the desired velocity magnitude. As shown in Figure 4 , the profile of Configuration (5) is located in the area where any single jet is no longer identifiable but where there is a more constant and homogeneous air velocity. A similar result characterises Configuration (6), with the only difference that the velocity magnitude is larger than Configuration (5). However, these configurations appear more suitable for underground cellar needs than the initial Configurations (1) and (2). Nevertheless, these results can be considered less satisfactory than those concerning Configurations (7) and (8), as shown comparing Figure 3 with Figure 5. In Configuration (7), the profile demonstrates to be more homogeneous and stable at $0.6 \mathrm{~m} / \mathrm{s}$, compared to the previous cases. In the profile, the velocity undergoes a small increase at the beginning and end areas of the pipe. These variations are very limited, just around $0.7 \mathrm{~m} / \mathrm{s}$, so that this configuration can be definitely considered similar to the sought results. The results of the two configurations are similar (Figure 5), since the profile presents
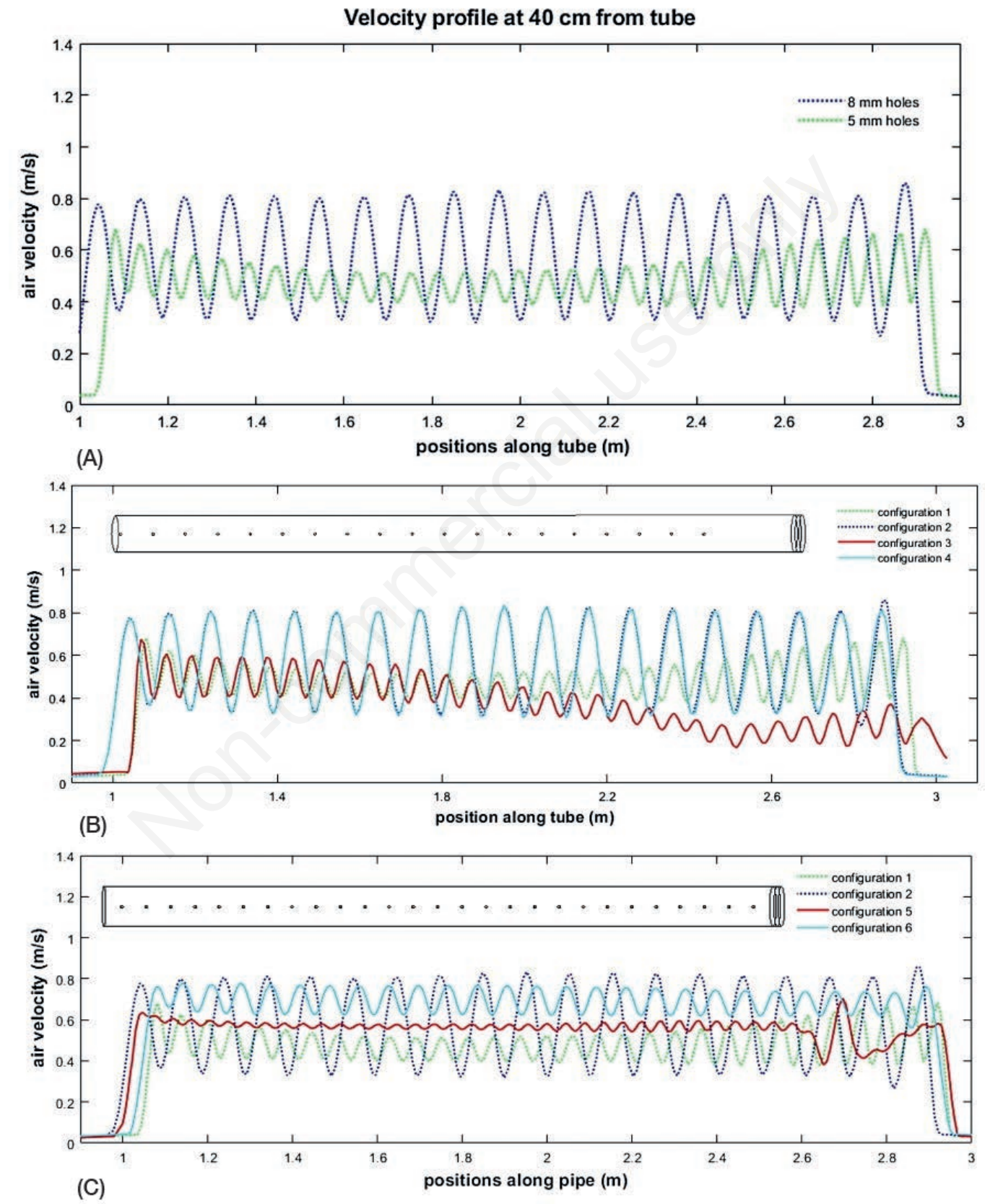

Figure 3. The top figure (A) is the comparison between the solutions with $5 \mathrm{~mm}$ and $8 \mathrm{~mm}$ holes. These are the two profile at $40 \mathrm{~cm}$ from the tube and represent the velocity magnitude starting from the end of the pipe to the fan. Figures $(B)$ and $(C)$ are the comparison of the previous configurations, represented with dashed lines, and the configurations $((1)$ and (2)) and whole lines are the configurations with $20 \mathrm{~cm}$ in addiction of tube. The dashed lines are the basic Configurations ((1) and (2)) and Configuration ((5) and (6) respectively, presented as whole lines. 
reduced oscillations and a more homogeneous trend, with a velocity magnitude settled around $0.8 \mathrm{~m} / \mathrm{s}$. In this case, there is a slight decrease in the magnitude in the initial part of the profile, but that does not lead to a significant variation in the trend.

Moreover, the distance of effectiveness of air jets has been investigated analysing the air velocity magnitude. Considering the target of the air ventilation system, the velocity values of greatest interest are those that are maintained in the range of $0.4 \mathrm{~m} / \mathrm{s}-1 \mathrm{~m} / \mathrm{s}$.

In order to identify at which distance both these limits have been exceeded, the velocity profiles of all configurations have been extrapolated every $2 \mathrm{~cm}$ of distance, starting from the air profile located $40 \mathrm{~cm}$ far from the pipe, and proceeding towards the external domain. In order to explain this methodology in the clearest way, the Configuration (7) has been taken as reference. The Figure 6 presents the air velocity profiles of the configuration analysed, starting from the velocity profiles at $40 \mathrm{~cm}$ and going towards and away from the pipe considered every $2 \mathrm{~cm}$. For a better representation, the air profiles going in the two different direc-
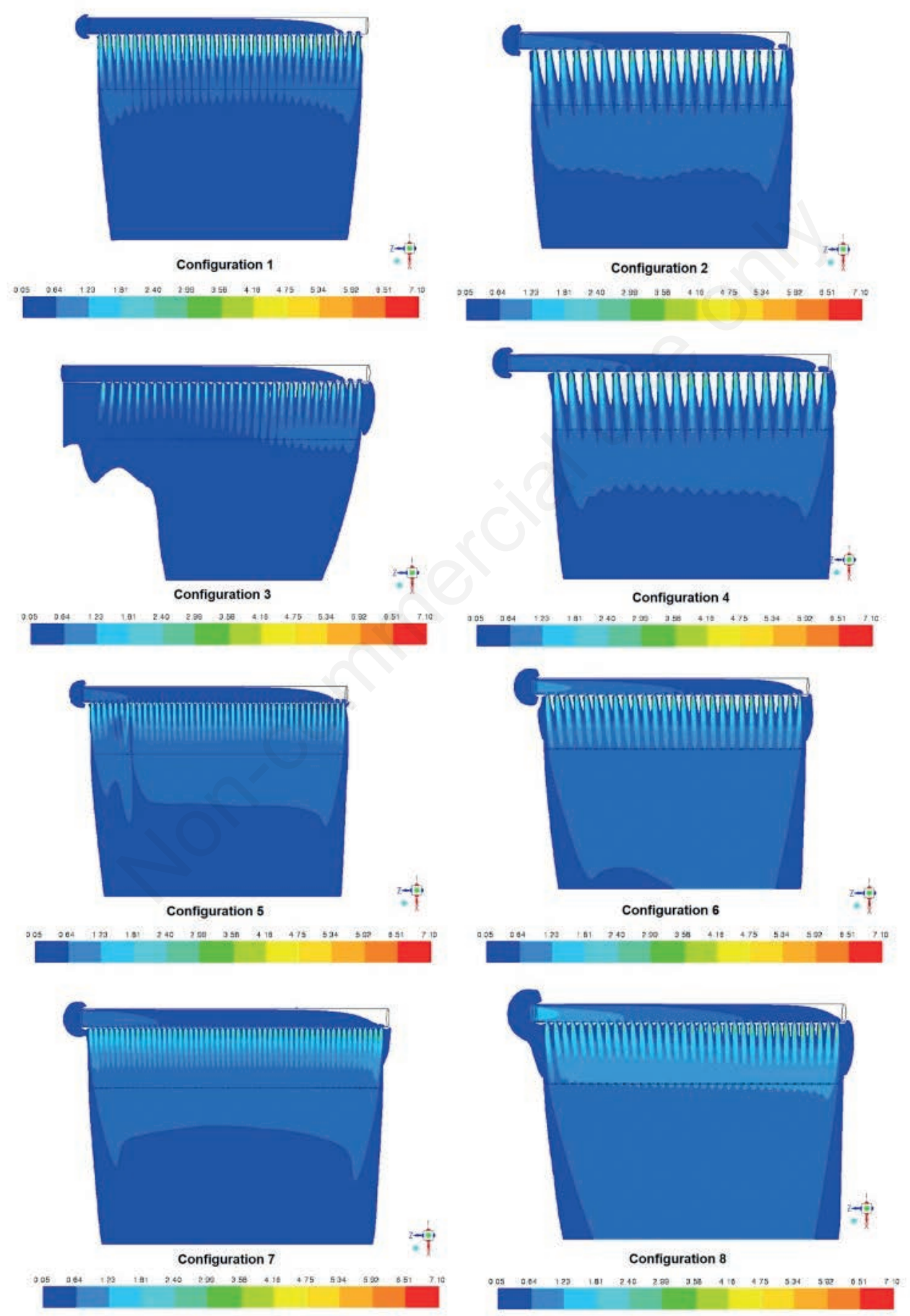

Figure 4. Contour maps of the configurations from (1) to (8). The air velocity profiles, at $40 \mathrm{~cm}$ of distance from the pipe, are depicted in black. 
tions are represented separately and only the profiles each 6 and 10 $\mathrm{cm}$ are presented in Figure 6. As visible in Figure 6A, the lower velocity limit of $0.4 \mathrm{~m} / \mathrm{s}$ is exceeded at a distance of $0.7 \mathrm{~m}$ from the tube. In fact, the profile at $0.7 \mathrm{~m}$ from the pipe is characterised in the central part by an air velocity lower than the limit. However, the air velocity has values lower than the limit all along the profile only at further distances. About the upper limit of $1 \mathrm{~m} / \mathrm{s}$, it is exceeded at a distance of $20 \mathrm{~cm}$, as visible in Figure 6B, all along the entire profile. In fact, in this position the air velocity profile has variations ranging from $1 \mathrm{~m} / \mathrm{s}$ to $0.7 \mathrm{~m} / \mathrm{s}$.

Then, it is clear that the area of action of the system is enclosed between $20 \mathrm{~cm}$ to $1 \mathrm{~m}$ of distance from the system. The same methodology has been applied to all the other configurations. Graphs including other configurations' profiles have not be reported for the sake of brevity, except the Configuration (8) towards- the-pipe profile due to its peculiarity. This configuration in fact exhibits a singular results: the distance from the tube - where the lower limit could be exceeded - cannot be easily identified in this way (in the subsequent calculations this distance has been defines), while the exceeding of the upper limit can be investigated, as done in Figure 7. The limit of $1 \mathrm{~m} / \mathrm{s}$ is overcome at a distance of $30 \mathrm{~cm}$ from the system, where the air velocity profile shows several peaks, located just in the first part of the velocity profile. The analyses so far described, have been collected and summarised in the identification of three parameters, in order to better compare all these configurations and to identify the most suitable solutions for underground cellars in general and for the paper case-study in particular. The parameters are: i) $\mathrm{v}_{\mathrm{m}}$ - mean velocity of the profile; ii) $\alpha$ - velocity variation along the profile; iii) $d_{\text {limit }}$ - limit distance of action of the system.

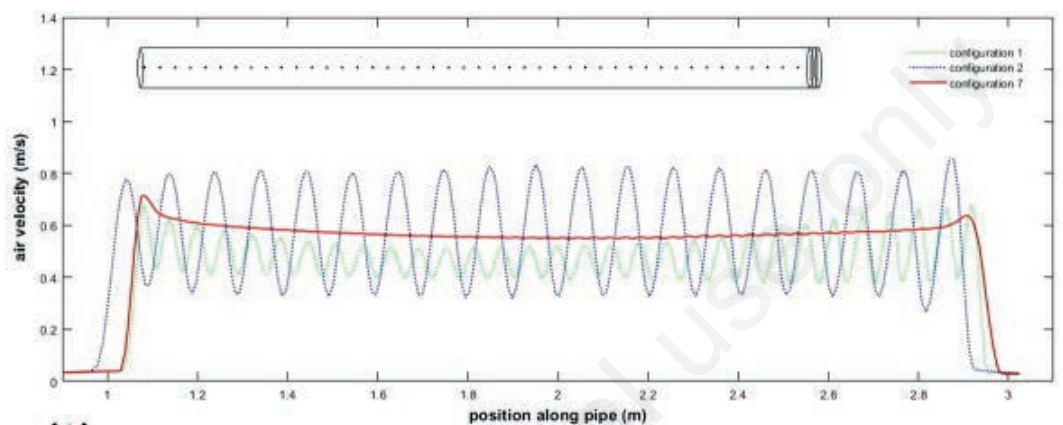

(A)

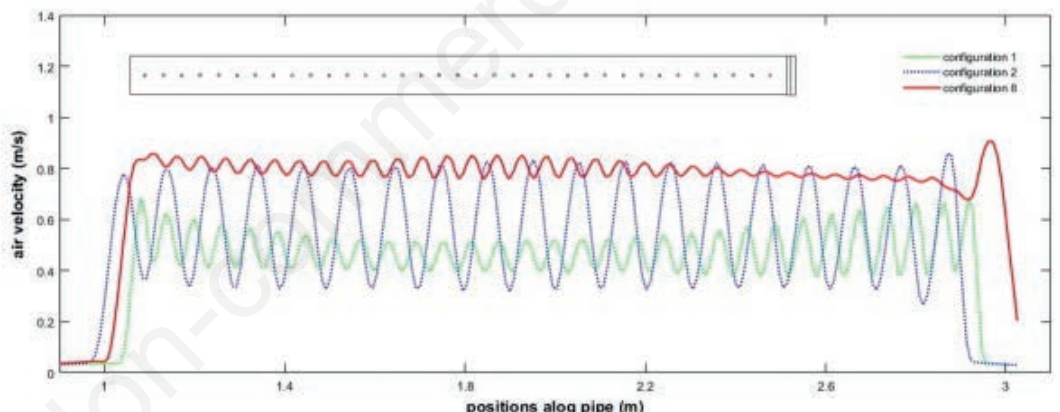

(B)

Figure 5. The top figure (A) is the comparison between the basic configurations (dashed lines) and the Configuration (7) (whole lines). The top figure $(\mathrm{B})$ is the comparison between the basic configurations (dashed lines) and the Configuration (8) (whole lines). The pipes are showed to understand that the profiles are from the end to the top of the tube.

Table 3. Important parameters for the differentiation of all analysed cases: $v_{\mathrm{m}}$ is the mean velocity of the profile at $40 \mathrm{~cm}$ from the pipe, $\sigma_{v}$ is the standard deviation of the velocity, $\alpha$ is the velocity variation along the profile, $\sigma_{\alpha}$ is the standard deviation related to the velocity variation and $d_{\text {limit }}$ is the limit distance of action of the system.

\begin{tabular}{lccccc} 
Configurations & $v_{m}(\mathrm{~m} / \mathrm{s})$ & $\mathrm{O}_{\mathrm{v}}(\mathrm{m} / \mathrm{s})$ & $\alpha(\mathrm{m} / \mathrm{s})$ & $\sigma_{\alpha}(\mathrm{m} / \mathrm{s})$ & $\mathrm{d} / \mathrm{imit}(\mathrm{m})$ \\
1 & 0.48 & \pm 0.11 & 0.17 & \pm 0.05 & 0.63 \\
2 & 0.6 & \pm 0.2 & 0.492 & \pm 0.016 & 1.08 \\
\hline 3 & 0.4 & \pm 0.11 & 0.143 & \pm 0.117 & 0.52 \\
4 & 0.53 & \pm 0.29 & 0.695 & \pm 0.033 & 1.05 \\
\hline 5 & 0.54 & \pm 0.10 & 0.172 & \pm 0.033 & 0.91 \\
6 & 0.75 & \pm 0.16 & 0.137 & \pm 0.015 & 1.03 \\
\hline 8 & 0.54 & \pm 0.12 & 0.015 & \pm 0.025 & 0.93 \\
\hline
\end{tabular}


In particular, the limit distance has been defined as the distance from the system where the air velocity starts to be under $0.4 \mathrm{~m} / \mathrm{s}$ $(\mathrm{v} \leq 0.4 \mathrm{~m} / \mathrm{s})$, taking in consideration three representative position along the tube (beginning, centre and end of the tube). Table 3 reports the three parameters and the standard deviations of $\mathrm{v}_{\mathrm{m}}\left(\sigma_{\mathrm{v}}\right)$ and $\alpha\left(\sigma_{\alpha}\right)$. At first, we analyse the configurations according to those parameters, under a general point of view. Later the parameters will be used to identify the most suitable configuration for the case-study. Taking into account the mean velocity and its standard deviation, all the configurations, except Configuration (3), present a velocity higher than $0.4 \mathrm{~m} / \mathrm{s}$, but Configuration (2) and Configuration (4) are characterised by a higher value of standard deviation. This fact is also confirmed by the value of $\alpha$, which are the highest values among all the configurations. About Configuration (3), a velocity equal to $0.4 \mathrm{~m} / \mathrm{s}$ is already reached at $40 \mathrm{~cm}$ from the tube.

However, this is an averaged value of a parameter that presents minimum and maximum peaks around 0.2 and $0.7 \mathrm{~m} / \mathrm{s}$. Instead, the value of $d_{\text {limit }}$ shows the distance where is present a peak of $0.4 \mathrm{~m} / \mathrm{s}$ and the other velocity values are already under this limit. Based on all parameters, Configurations (5), (6), (7) and (8) demonstrates effective air velocity magnitudes, low values of velocity variation along the profile and also the greater distances of action. However, among these, Configurations (7) and (8) exhibit lower velocity variation $\alpha$ and small related standard deviation entailing to be the more stable profiles compared to all the other cases. About these
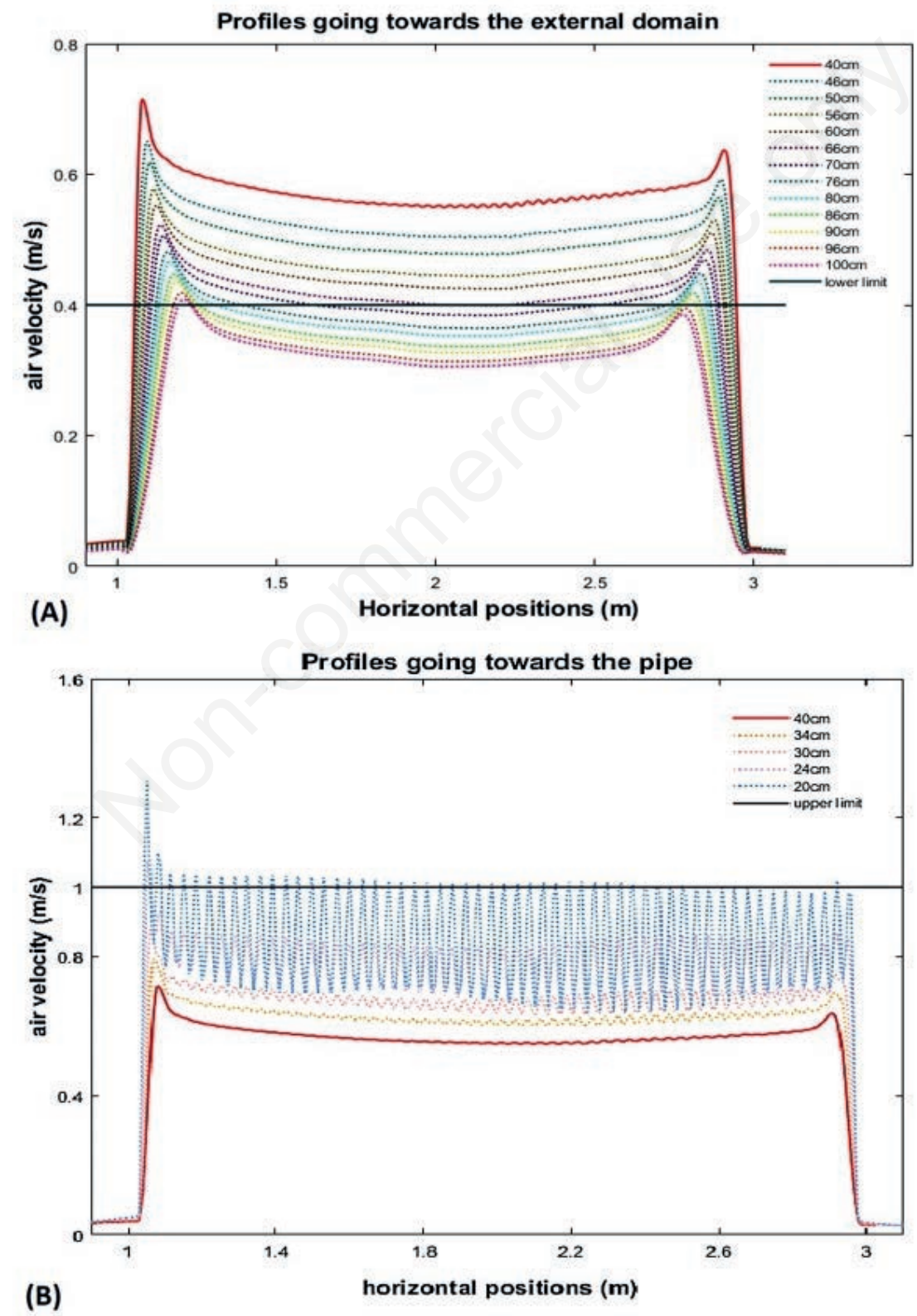

Figure 6. The first plot (A) presents the trend of the velocity profiles, increasing the distance from the pipe in Configuration 7 . The second plot (B) shows the same situation but about the profiles with a decreasing distance from the tube. 
two cases, the air flows have been analysed more in detail as shown in Figures 6 and 7, where the area of action of Configuration (7) has been restricted between $20 \mathrm{~cm}-1.06 \mathrm{~m}$ from the system and between $30 \mathrm{~cm}-1.45 \mathrm{~m}$ for Configuration (8). These information and related consideration are essential to evaluate the positions of the system inside the room.

The Configurations (2), (3) and (4) prove to return worse performances compared to the other solutions. Configuration (7) and (8) are the more stable and with the longer range of action. In the case-study systems designed according to Configuration (7) and (8), the ventilation system can be placed even farther from the barriques (for example in the ceiling) due to long range of effectiveness and strong stability. For this kind of application, also systems designed according to Configuration (5) and (6) can be applied with a lower air velocity stability than the previous cases. On the contrary, Configuration (1) can be a suitable solution to be placed between the barriques and the wall thank to $\mathrm{v}_{\mathrm{m}}$ close to lower limit and a good value of $\alpha$.

This fact could lead to an increasing dimension of the conduct, of the holes and a more powerful fan. Even though the results of this paper cannot be taken into account for a direct application on livestock barns, the proposed methodology can be a useful tool for specific studies in this sector.

On the contrary, referring to the greenhouse needs, the present system could be adopted in these constructions. Considering the resulting air velocity, in fact, this system could be used in a greenhouse for plant ventilation. The literature shows that in the cultivation area the optimal range of ventilation is $0.5-0.3 \mathrm{~m} / \mathrm{s}$ (Hanan, 2017; Santolini et al., 2018) but not always this range can be maintained by the natural ventilation. This system could cover this lack of ventilation efficiency. Another relevant aspect for alternative usages of this system is the orientations of the jets in relation to the environmental parameter required in the room. In this study, the air jets have been considered perpendicular to the barrels, but in few situations, changing the air velocity direction and/or providing a wider-angle range could return better performances. This aspect too requires specific studies for the specific needs.

\section{Conclusions}

Considering the important role of the environmental conditions, the control systems are rarely applied in wine cellar. Usually these rooms are naturally ventilated, and this solution presents several issues especially, as already widely explained previously.

According to these, the design and test process of a smart ventilation system have been conducted, which would act as a support for natural ventilation, for small or medium wine farms. In particular, a first set up of the system has been created and a 3D CFD model of it has been performed. The model has been validated by means of experimental data. Then, several further configurations of the systems have been analysed and compared, defining the best ones based on the system goals. Based on the defined parameters, the best performing configurations are characterised by: i) holes of $0.5 \mathrm{~mm}$ of diameter, spaced $3 \mathrm{~cm}$ from each other; ii) holes of 0.8 $\mathrm{mm}$ of diameter, spaced $4.8 \mathrm{~cm}$ from each other.

The CFD approach has given the chance to observe the behaviour of each system hypothesised, identifying positive and negative aspects in relation to the desired results, based on specific parameters. These parameters can be useful indicators in any case for the identification of the best configuration based on the wine cellar characteristics and necessities. Finally, the presented methodology has proved to be an important design tool to validate, test and optimise the ventilation system, avoiding the necessity to build any new system, saving time and materials. This approach has allowed also the investigation of other useful aspects for the further system implementation, such as the zones of air velocity, within the defined magnitude range. All these analyses can be considered essential for a proper placement of the system inside the room. In the agricultural sector, other buildings (such as livestock barns and greenhouses) can require a ventilation system able to perform a constant, distributed, low-velocity flow. Referring to the livestock sector, the ventilation is usually necessary for the animal welfare (Carpenter, 1972; Rong et al., 2016), however the parameters of this ventilation are remarkably different from those ones performed by the system studied in this work. In particular, air flow and velocities should be



Figure 7. The plot presents the trend of the velocity profiles, decreasing the distance from the pipe, in order to identify when the limit of $1 \mathrm{~m} / \mathrm{s}$ is passed. 
definitely higher, over $1 \mathrm{~m} / \mathrm{s}$, in a range between 2-3 m/s (Wang et $a l ., 2018)$. Starting from these results, it would be interesting to investigate how the implementation of the system could affect the global air flow distribution inside the wine cellar. Future works will assess the effectiveness of this system in a cellar in terms of temperature and humidity uniformity.

\section{References}

Avramidis S., Siau J.F. 1987. An investigation of the external and internal resistance to moisture diffusion in wood. Wood Sci. Technol. 21:249-56.

Barbaresi A., De Maria F., Torreggiani D., Benni S., Tassinari P. 2015a. Performance assessment of thermal simulation approaches of wine storage buildings based on experimental calibration. Energ. Build. 103:307-16.

Barbaresi A., Torreggiani D., Benni S., Tassinari P. 2014. Underground cellar thermal simulation: Definition of a method for modelling performance assessment based on experimental calibration. Energ. Build. 76:363-72.

Barbaresi A., Torreggiani D., Benni S., Tassinari P. 2015b. Indoor air temperature monitoring: A method lending support to management and design tested on a wine-aging room. Build. Environ. 86:203-10.

Bartzanas T., Kacira M., Zhu H., Karmakar S., Tamimi E., Katsoulas N., Lee I.B., Kittas C. 2013. Computational fluid dynamics applications to improve crop production systems. Compag. 93:151-67.

Benni S., Torreggiani D., Barbaresi A., Tassinari P. 2013. Thermal performance assessment for energy-efficient design of farm wineries. Trans. ASABE 56:1483-91.

Boulton R.B., Singleton V.L., Bisson L.F., Kunkee R.E. 1999. Principles and practices of winemaking. Springer, Berlin, Germany.

Brundrett E. 1990. Prediction of airflow with swirl in perforated polyethylene tubes. J. Fluids Engine. 112:447-54.

Carpenter G.A. 1972. The design of permeable ducts and their application to the ventilation of livestock buildings. J. Agricult. Engine. Res. 17:219-30.

De la Torre-Gea G., Soto-Zarazùa M.G., Lopez-Crùz I., Pacheco Torres I., Rico-Garcìa E. 2011. Computational fluid dynamics in greenhouses: A review. Afr. J. Biotechnol. 10:17651-62.

De Rosis A., Barbaresi A., Torreggiani D., Benni S., Tassinari P. 2014. Numerical simulations of the airflows in a wine-aging room: A lattice Boltzmann-Immersed Boundary study. Compag. 109:261-70.

Fluent Inc. 2006. FLUENT 6.3 User's Guide. New York, NY, USA.

Geyrhofer A.F., Weingartmann H., Mandl K., Schattauer D. 2011. Measurements of air flow in the wine cellar. Mitteilungen Klosterneuburg 61:76-81.

Hanan J.J. 2017. Greenhouses-advanced technology for protected horticulture. Ed. CRC Press, Boca Raton, FL, USA.

Magnini M., Pulvirenti B., Thome J. 2016. Characterisation of the velocity fields generated by flow initialisation in the cfd simulation of multiphase flows. Appl. Math. Model. 40:6811-30.

Mazarron F.R., Cañas I. 2009. Seasonal analysis of the thermal behaviour of traditional underground wine cellars in Spain. Renew. Energ. 34:2484-92.

Mondaca M., Choi C.Y. 2017. A computational fluid dynamics model of a perforated polyethylene tube ventilation system for dairy operations. vol. 59. pp 1585-1594 in 2017 Spokane, Washington, July 16-19, 2017.

Negrè E., Françot P. 1965. Manuel pratique de vinification et de conservation des vins. Flammarion ed., Les Angles, Paris, France.

Rong L., Nielsen P.V., Bjerg B., Zhang G. 2016. Summary of best guidelines and validation of cfd modeling in livestock buildings to ensure prediction quality. Comput. Electron. Agric. 121:180-90.

Ruiz De Adana M., Lopez L.M., Sala J.M. 2005. A Fickian model for calculating wine losses from oak casks depending on conditions in ageing facilities. Appl Thermal Engine. 25:709-18.

Santolini E., Pulvirenti B., Benni S., Barbaresi L., Torreggiani D., Tassinari P. 2018. Numerical study of wind-driven natural ventilation in a greenhouse with screens. Comput. Electron. Agric. 149:41-53.

Shih T.-H., Liou W.W., Shabbir A., Yang Z., Zhu J. 1995. A new k$\varepsilon$ eddy viscosity model for high reynolds number turbulent flows. Comput. Fluids 24:227-38.

Simeray J., Mandin D., Mercier M., Chaumont J.P. 2001. Survey of viable airborne fungal propagules in French wine cellars. Aerobiologia 17:19-24.

Togores H.J. 2003. Tratado de enologìa. Editiones Mundi-Prensa, Madrid, Spain, p 1423.

Vogt E. 1971. Fabricacion de vinos (Winemaking). Editorial Acribia, Zaragoza, Spain.

Wang X., Zhang G., Choi C. 2018. Evaluation of a precision airsupply system in naturally ventilated freestall dairy barns. Biosyst. Eng. 175:1-15. 